enrolled in the Västerbotten Intervention Project (a subgroup of the Northern Sweden Health and Disease Cohort). Postload glucose concentration was measured in capillary plasma $2 \mathrm{~h}$ after an oral glucose tolerance test with $75 \mathrm{~g}$ glucose, in accordance with WHO standards.

After correction for random variations in glucose concentration over time, women with elevated fasting glucose and postload glucose concentrations were at considerably increased overall risk of cancer (relative risks 1.75 and 1.63, respectively). There was no significant increase in overall cancer risk in men. Combined data for both men and women with high levels of fasting glucose showed a markedly increased risk of cancers of the pancreas and urinary tract, and also for malignant melanomas. Adjustment for BMI had only minor effects on cancer risk, and there was no significant difference in hyperglycemia-associated cancer risk between smokers and nonsmokers.

The authors conclude that their results agree with those from previous studies, and they suggest that a lifestyle conducive to decreased plasma glucose levels might reduce overall cancer risk among individuals with normal BMI, as well as overweight and obese individuals traditionally considered at high hyperglycemia-related cancer risk.

Original article Stattin P et al. (2007) Prospective study of hyperglycemia and cancer risk. Diabetes Care 30: 561-567

\section{Reducing television viewing time might help to prevent diabetes}

Sedentary behavior is known to increase the risk of various chronic diseases, including type 2 diabetes and the metabolic syndrome. Dunstan and colleagues analyzed data from a large, population-based, Australian study to assess the relationship between a major sedentary leisuretime activity—television viewing —and measures of glycemia.

Fasting plasma glucose and $2 \mathrm{~h}$ postchallenge glucose levels were measured in 3,781 men and 4,576 women (aged 36-91 years) with no history of diabetes. Insulin sensitivity and $\beta$-cell function were calculated using the homeostasis model assessment algorithm. Total television viewing time per week was estimated from interviewer-administered questionnaires.

After adjustment for age and potential confounding variables (including body habitus, physical activity and diet), prolonged television viewing time was associated with increased $2 \mathrm{~h}$ postchallenge glucose levels, fasting insulin levels and $\beta$-cell function, and reduced insulin sensitivity, in female participants. No significant associations were found between television viewing time and glycemic measures in men, or between television viewing time and fasting plasma glucose levels in either men or women.

The authors suggest that the adverse effect of prolonged television viewing might be caused by disruption of normal metabolic functions. The difference between sexes might be explained by metabolic or behavioral differences. The authors conclude that, besides promoting physical activity, health professionals should also advise people-especially women-to minimize their television viewing and other sedentary behaviors to reduce their risk of type 2 diabetes and cardiovascular disease.

Original article Dunstan DW et al. (2007) Association of television viewing with fasting and 2 -h postchallenge plasma glucose levels in adults without diagnosed diabetes. Diabetes Care 30: 516-522

\section{New genetic risk factors identified for type 2 diabetes}

The study of genetic risk factors for type 2 diabetes mellitus has experienced rapid development since the advent of new molecular biological methods that enabled systematic analysis of genetic polymorphisms in the human genome. Sladek and colleagues assessed 392,935 single-nucleotide polymorphisms for their potential association with type 2 diabetes in a French cohort.

The study included 694 diabetic individuals (cases) who developed type 2 diabetes before the age of 45 years, had at least one affected first-degree relative and a BMI $<30 \mathrm{~kg} / \mathrm{m}^{2}$, and 654 nondiabetic (control) individuals with fasting plasma glucose levels $<5.7 \mathrm{mmol} /$. The researchers used high-density arrays to assess the genotype of each participant and, among the polymorphisms with the largest differences in frequency between the two groups, they found eight for which this difference was replicated in an additional sample of 2,617 cases and 2,894 controls.

The eight variants associated with an increased risk of type 2 diabetes were found at five loci, one of which has been identified 